\title{
Intrapulmonic Cavity or Necrosis on Baseline CT Scan Serves as an Efficacy Predictor of Anti-PD- (L)I Inhibitor in Advanced Lung Squamous Cell Carcinoma
}

\author{
Tao Lu', ${ }^{1}$ \\ Longfeng Zhang ${ }^{2, *}$ \\ Mingqiu Chen $\mathbb{D}^{3}$ \\ Xiaobin Zheng $\left(\mathbb{D}^{2}\right.$ \\ Kan Jiang ${ }^{2}$ \\ Xinlong Zheng ${ }^{2}$ \\ Chao $\mathrm{Li}^{4}$ \\ Weijin $\mathrm{Xiao}^{4}$ \\ Qian Miao ${ }^{2}$ \\ Shanshan Yang ${ }^{2}$ \\ Gen $\operatorname{Lin}^{2}$ \\ 'Department of Radiology, Fujian Medical \\ University Cancer Hospital, Fujian \\ Cancer Hospital, Fuzhou, People's \\ Republic of China; ${ }^{2}$ Department of \\ Thoracic Oncology, Fujian Medical \\ University Cancer Hospital, Fujian \\ Cancer Hospital, Fuzhou, People's \\ Republic of China; ${ }^{3}$ Department of \\ Thoracic Radiation Oncology, Fujian \\ Medical University Cancer Hospital, \\ Fujian Cancer Hospital, Fuzhou, People's \\ Republic of China; ${ }^{4}$ Department of \\ Pathology, Fujian Medical University \\ Cancer Hospital, Fujian Cancer Hospital, \\ Fuzhou, People's Republic of China \\ *These authors contributed equally to \\ this work
}

Background: Predictive markers for guidance and monitoring of immunotherapy in lung squamous cell carcinoma (LSCC) are an interesting topic but have yet to be fully explored. A primary characteristic of LSCC is tumor necrosis that results in extensive immune suppression in patients. We sought to assess whether tumor necrosis or cavity on baseline CT could effectively predict the efficacy of immune checkpoint inhibitors (ICIs) in advanced LSCC.

Methods: Advanced LSCC cases undergoing pre-treatment chest CT imaging and receiving ICIs were retrospectively collected. All CT images were reviewed by an independent chest radiologist blinded to any previous diagnosis to confirm morphological alterations in necrosis or cavity. We performed Logistic regression and developed Cox proportional hazards models to assess the predictive performance of baseline necrosis or cavity characteristics in advanced LSCC. Survival estimates were observed using Kaplan-Meier curves.

Results: Ninety-three patients were eligible for analysis, predominantly consisting of patients with ECOG performance status of 0 or $1(97.8 \%)$, male patients $(95.7 \%)$, and heavy smokers (92.5\%). Intrapulmonic necrosis or cavity on CT scan was present in $52.7 \%$ of all patients. Generally, the objective response rate (ORR) in patients with necrosis or cavity to ICI treatment was significantly worse versus those without $(30.6 \%$ vs $54.5 \%, p=$ 0.020 ), with the subgroup ORRs as follows: ICI monotherapy (necrosis vs non-necrosis: $10.0 \%$ vs $36.8 \%, p=0.047)$ and ICI combination therapy $(44.8 \%$ vs $68.0 \%, p=0.088)$. Multivariable analysis identified intrapulmonic necrosis or cavity at baseline as a major risk factor for advanced LSCC (HR 4.042, 95\% CI1.149-10.908, $p=0.006$ ). Multivariate Cox analysis showed that baseline necrosis or cavity and ICI monotherapy were unfavorable factors for progression-free survival (HR 1.729; 95\% CI1.203-2.484, $p=0.003$ ).

Conclusion: LSCC patients with intrapulmonic cavity or necrosis on baseline CT scan may respond poorly to anti-PD-(L)1-treatment, monotherapy and combination therapy alike.

Keywords: lung squamous cell carcinoma, immune-checkpoint inhibitor, predictive marker, necrosis, cavity

\section{Introduction}

Department of Thoracic Oncology, Fujian Medical University Cancer Hospital,

Fujian Cancer Hospital, Fuzhou, People's Republic of China

Email fjzllg133@fjzlhospital.com
Immune checkpoint inhibitors (ICIs) have transformed our approach for non-small cell lung cancer (NSCLC) management. ${ }^{1}$ A selection of biomarkers, programmed cell death-ligand 1 (PD-L1), ${ }^{2-6}$ tumor mutational burden (TMB) ${ }^{7,8}$ mismatch repair 
deficiency/microsatellite instability-high ${ }^{9-11}$ and beyond, have exhibited excellent utilities in a considerable share of beneficiaries.

Adenocarcinoma and squamous cell carcinoma (SCC), featuring distinct pathogenesis, biological processes, and immune microenvironments, are two major histopathological components and NSCLC subtypes. Among others, lung adenocarcinoma seems to have a better prognosis following the identification of predictors of response to ICI treatment. However, efficacy predictor research for SCC has only produced more disappointing results due to lack of druggable driver gene. ${ }^{12}$ PD-L1 IHC expression is the only biomarker currently approved for clinical use. ${ }^{3-}$ ${ }^{6}$ Whether there is a histology-specific expression of PDL1 between SCC versus non-SCC is still under debate. For example, a large observational, retrospective study of LSCC cases showed no relationship between PD-L1 TPS $\geq 50 \%$ and overall survival of patients receiving the firstline pembrolizumab. ${ }^{13}$ It is too early to make a definitive conclusion about the clinical utility of PD-L1 expression as a predictor of response to ICI treatment in LSCC.

Tumor necrosis is a typical characteristic of the vast majority of malignancies. ${ }^{14,15}$ Foci of cell death are frequently observed in core regions of solid tumors as a result of inadequate vascularization and subsequent metabolic stresses, such as hypoxia and nutrient deprivation. ${ }^{16,17}$ Although studies over the past decades have explored the functions and consequences of cellular demise and elucidated several essential cell death pathways, the exact role of tumor necrosis in tumor development and metastasis remains elusive. ${ }^{15}$ Unlike apoptosis, by which tumor cells are rapidly removed by host macrophages without harming the membrane of normal cells, necrosis may unleash host immune suppression via consistently releasing immunesuppressive inflammatory molecules and recruiting inflammatory cells in the tumor microenvironment. ${ }^{18-21} \mathrm{~A}$ recent study found that enhanced potassium outflow from necrotic tumor cells and the resultant high potassium concentration led to impairing $\mathrm{T}$ cell receptor (TCR)-driven Akt-mTOR phosphorylation and effector programmes. Potassiummediated suppression of Akt-mTOR signalling and $\mathrm{T}$ cell function is dependent upon the activity of the serine/threonine phosphatase PP2A3,4, thus can inhibit CD4 and CD8 T cell activities, which are critical for anti-tumor immunity. ${ }^{22}$

We hypothesized that intrapulmonic cavity or necrosis was associated with worse outcomes and lower response rates to ICIs. Chest computed tomography (CT) is a valid approach for monitoring response to treatment in lung cancer, and the presence of necrosis or cavity on CT scan has been numerously reported. So, the fundamental objectives of our study were to explore whether baseline cavity or necrosis on baseline CT scan were satisfactory efficacy predictors of response to ICIs in advanced lung squamous cell carcinoma (LSCC).

\section{Methods}

\section{Patients}

We reviewed the medical records of all patients admitted to Fujian Medical University Cancer Hospital, Fujian Cancer Hospital between April 2015 and December 2020. Those diagnosed with recurrent or advanced LSCC and underwent contrast-enhanced chest CT imaging at baseline and received ICI treatment were included for further analysis. These patients must be followed for at least one time. The following clinicopathological features of each patient were extracted: age at ICI treatment, gender, smoking status at admission (heavy smokers individuals who had at least 20 pack-years of smoking; light smokers individuals who had less than 20 pack-years of smoking), Eastern Cooperative Oncology Group (ECOG) performance score (PS), the sum of diameters of all measurable tumor lesions, brain, liver, and bone metastases, lactate dehydrogenase (LDH), neutrophil-to-lymphocyte ratio (NLR), and PD-L1 tumor proportion score (22C3 pharmDx assay). NLR was calculated by dividing the absolute neutrophil count by the absolute lymphocyte count measured in peripheral blood at baseline.

Tumor response was assessed using CT and the Response Evaluation Criteria in Solid Tumor (RECIST, version 1.1). ${ }^{23}$ Objective response rate (ORR) was calculated as the proportion of patients who showed the best response, using a complete response (CR) or partial response (PR), as appropriate. Disease control rate (DCR) was defined as the number of patients with the best response (CR or PR) or stable disease (SD) divided by the total number of subjects. Progression-free-survival (PFS) represented the duration between initiation of treatment and clinical or radiographic progression or death from any cause. Informed consent was waived for this retrospective study.

\section{CT Scan}

CT data were converted into Digital Imaging and Communications in Medicine (DICOM) format. Baseline 
chest CT with $5 \mathrm{~mm}$ and $2 \mathrm{~mm}$ collimations was performed for lung cancer screening. Most CT images were photographed using both mediastinal (level, $40 \mathrm{HU}$; width, $400 \mathrm{HU}$ ) and lung (level, -600 HU; width, $1600 \mathrm{HU}$ ) window settings. Imaging features were defined according to recommendations of the Nomenclature Committee of the Fleischner Society (NCFS). ${ }^{24}$ In contrast-enhanced chest $\mathrm{CT}$, necrosis is referred to as an intratumoral area larger than $5 \mathrm{~mm}$ in diameter with a 10-to-30 HU change and no enhancement greater than $10 \mathrm{HU}$ after contrast administration. The pulmonary cavity was unanimously described as "gas-filled space within a zone of pulmonary consolidation or a mass or nodule, seen as a lucency or low-attenuation area." Chest imaging features, intrapulmonic necrosis and cavity in particular, were reviewed by an independent radiologist blinded to patient's outcomes to ICIs.

\section{Statistical Analysis}

The Chi-squared test and logistic regression analysis were applied to assess clinical parameters associated with ICI efficacy. The receiver operating characteristic (ROC) curves, area under the curve (AUC) of the ROC, sensitivity, specificity, and likelihood ratios were calculated to determine cutoffs of the summed diameters of all measurable tumor lesions, LDH, and NLR to discriminate clinical benefit responders $(\mathrm{CR} / \mathrm{PR})$ from non-responders $(\mathrm{SD} / \mathrm{PD})$. We utilized Kaplan-Meier curves to estimate associations of these clinical characteristics with PFS, with significance assessed using the Log rank test. The hazard ratio (HR) with its $95 \%$ confidence interval (CI) was calculated for survival prediction based on univariate Cox proportional hazard models. Two-sided P-values of $<0.05$ were considered statistically significant. A multivariate Cox proportional hazards model was developed by incorporating covariates with a $p<0.05$ on univariate analysis. All statistical analyses were performed after the database was locked on March 1, 2021, using the SPSS version 20 (IBM Corp., Chicago, IL).

\section{Results}

\section{Patient Characteristics and ICI Treatment}

We identified a total of 106 advanced LSCC patients who had received ICI therapy, 93 of whom had complete baseline CT imaging data. Their clinicodemographic profile was summarized in Table 1. Intrapulmonic necrosis or cavity was identified in $52.7 \%$ of all LSCC patients, particularly in those with a good ECOG PS ( 0 or 1 in $97.8 \%$ of cases), male patients $(95.7 \%)$, and heavy smokers $(92.5 \%)$.

The sum of diameters of all measurable lesions describes the magnitude of the tumor burden. Comparisons showed that patients with an intrapulmonic necrosis or cavity exhibited a larger summed diameter versus those without necrosis or cavity. And they also exhibited a higher incidence of bone metastases at baseline (Table 1). More LSCC patients with necrosis or cavity expressing PD-L1 (TPS $\geq 1 \%$ ) versus non-necrosis individuals $(30.6 \%$ vs $18.2 \%)$, without statistical significance. LDH and NLR, which represent potential biomarkers for the prediction of the efficacy of PD-(L)1 checkpoint inhibitors against NSCLC, were also examined. In this study, the proportions of high baseline LDH ( $>250 \mathrm{IU} / \mathrm{L})$ and NLR (>3.0) were not significantly different between the two groups.

Additionally, 54 patients (58.1\%) receiving concurrent chemotherapy or radiotherapy were assigned to the combination subgroup. Patients treated with the first-, second-, and third-line ICIs or beyond were accounted for $46.2 \%, 38.7 \%$ and $15.1 \%$ of all patients. The median duration of the entire ICI administrations was 6 weeks (range, 1-45 weeks).

\section{Response}

At the time of data cutoff, response assessment for an ORR to ICIs was available for all patients, which was 41.9\%. The disease control rate was 64.5\%. Subgroup comparisons showed that patients treated with monotherapy achieved a lower ORR than the combination group (23.1\% vs $55.6 \%, p=0.004)$. We then employed analysis to assess ORR, taking into account a significant difference between treatment patterns, with treatment pattern as a stratifying factor. In the monotherapy subgroup, patients with intrapulmonic necrosis or cavity at baseline exhibited a lower ORR versus non-necrosis cases (10.0\% vs $36.8 \%$, $p=0.047$ ) (Table 2). The comparison in the combination group revealed an insignificant rise in ORR in necrosis patients versus non-necrosis ones $(68.0 \%$ vs $44.8 \%$, $p=0.088$ ) (Table 2).

Next, we validated these findings using logistic regression analysis. Still, ORRs to ICI treatment was significantly associated with baseline necrosis or cavity in the lung and treatment patterns $(\mathrm{OR}=4.402$, 95\% CI 1.149$10.908, p=0.006$ ) (Table 3 ), but not with other clinical characteristics, as indicated by both univariate and multivariate analyses. For continuous variables such as the 
Table I Patient Characteristics

\begin{tabular}{|c|c|c|c|c|c|}
\hline & & \multirow[t]{2}{*}{ Total } & \multicolumn{2}{|c|}{ Cavity and/or Necrosis N (\%) } & \multirow[t]{3}{*}{ p-value } \\
\hline & & & Positive & Negative & \\
\hline & & $\mathbf{N}(\%)$ & $\mathbf{N}(\%)$ & $\mathbf{N}(\%)$ & \\
\hline Gender & $\begin{array}{l}\text { Male } \\
\text { Female }\end{array}$ & $\begin{array}{c}89 \text { (95.7\%) } \\
4(4.3 \%)\end{array}$ & $\begin{array}{c}4 \mathrm{I}(93.2 \%) \\
3(6.8 \%)\end{array}$ & $\begin{array}{c}48(98.0 \%) \\
\text { I (2.0\%) }\end{array}$ & 0.534 \\
\hline Smoking & $\begin{array}{l}\text { Light or never } \\
\text { Heavy }\end{array}$ & $\begin{array}{c}7(7.5 \%) \\
86(92.5 \%)\end{array}$ & $\begin{array}{c}3(6.8 \%) \\
41(93.2 \%)\end{array}$ & $\begin{array}{c}4(8.2 \%) \\
45(91.8 \%)\end{array}$ & 0.806 \\
\hline Age & $\overline{\mathrm{X}} \pm \mathrm{SD}$ & $61.3 \pm 8.8$ & $60.8 \pm 9.1$ & $61.8 \pm 8.7$ & 0.565 \\
\hline ECOG PS & $\begin{array}{l}0 \\
1 \\
2\end{array}$ & $\begin{aligned} 1 & (1.1 \%) \\
90 & (96.8 \%) \\
2 & (2.2 \%)\end{aligned}$ & $\begin{array}{c}\text { I }(2.3 \%) \\
42(95.5 \%) \\
\text { I }(2.3 \%)\end{array}$ & $\begin{array}{c}0 \text { (0.0\%) } \\
48(98.0 \%) \\
\text { I (2.0\%) }\end{array}$ & 0.567 \\
\hline Sum of tumor lesion diameters & $\overline{\mathrm{X}} \pm \mathrm{SD}$ & $67.7 \pm 38.8$ & $47.5 \pm 23.1$ & $85.7 \pm 41.1$ & $<0.001 *$ \\
\hline Brain metastases & $\begin{array}{l}\text { Presence } \\
\text { Absence }\end{array}$ & $10(10.8 \%)$ & $3(6.8 \%)$ & 7 (14.3\%) & 0.205 \\
\hline Liver metastases & $\begin{array}{l}\text { Presence } \\
\text { Absence }\end{array}$ & $13(14.0 \%)$ & $8(18.2 \%)$ & $5(10.2 \%)$ & 0.405 \\
\hline Bone metastases & $\begin{array}{l}\text { Presence } \\
\text { Absence }\end{array}$ & $25(26.9 \%)$ & $6(13.6 \%)$ & $19(38.8 \%)$ & $0.009 *$ \\
\hline LDH & $\begin{array}{l}\bar{X} \pm S D \\
\leq 250 \mathrm{IU} / \mathrm{L} \\
>250 \mathrm{IU} / \mathrm{L}\end{array}$ & $\begin{array}{c}244.1 \pm \mid 36.0 \\
70(75.3 \%) \\
23(24.7 \%)\end{array}$ & $\begin{array}{c}212.8 \pm 54.9 \\
35(79.5 \%) \\
9(20.5 \%)\end{array}$ & $\begin{array}{c}272.2 \pm 176.2 \\
35(71.4 \%) \\
14(28.6 \%)\end{array}$ & $\begin{array}{l}0.035^{*} \\
0.506\end{array}$ \\
\hline NLR & $\begin{array}{l}\bar{X} \pm S D \\
\leq 3.0 \\
>3.0\end{array}$ & $\begin{array}{c}3.5(2) \\
44(47.3 \%) \\
49(52.7 \%)\end{array}$ & $\begin{array}{c}3.3(1.9) \\
25(56.8 \%) \\
19(43.2 \%)\end{array}$ & $\begin{array}{c}3.8(2.0) \\
19(38.8 \%) \\
30(61.2 \%)\end{array}$ & $\begin{array}{l}0.272 \\
0.125\end{array}$ \\
\hline PD-LI TPS & $\begin{array}{l}\text { Done } \\
0(0 \%) \\
1(1-49 \%) \\
2(>50 \%) \\
\text { Not done }\end{array}$ & $\begin{array}{c}9(28.1 \%) \\
15(46.9 \%) \\
8(25.0 \%) \\
61(65.6 \%)\end{array}$ & $\begin{array}{c}3(27.3 \%) \\
5(45.5 \%) \\
3(27.3 \%) \\
33(75.0 \%)\end{array}$ & $\begin{array}{c}6(28.6 \%) \\
10(47.6 \%) \\
5(23.8 \%) \\
28(57.1 \%)\end{array}$ & 0.345 \\
\hline Treatment model & $\begin{array}{l}\text { Monotherapy } \\
\text { Combination therapy }\end{array}$ & $\begin{array}{l}39(41.9 \%) \\
54(58.1 \%)\end{array}$ & $\begin{array}{l}19(43.2 \%) \\
25(56.8 \%)\end{array}$ & $\begin{array}{l}20(40.8 \%) \\
29(59.2 \%)\end{array}$ & 0.983 \\
\hline Treatment line & $\begin{array}{l}\text { Ist } \\
\text { 2nd } \\
\geq 3 \text { rd }\end{array}$ & $\begin{array}{l}43(46.2 \%) \\
36(38.7 \%) \\
14(15.1 \%)\end{array}$ & $\begin{array}{c}17(38.6 \%) \\
22(50.0 \%) \\
5(11.4 \%)\end{array}$ & $\begin{array}{l}26(53.1 \%) \\
14(28.6 \%) \\
9(18.4 \%)\end{array}$ & 0.102 \\
\hline $\mathrm{ICl}$ agent & $\begin{array}{l}\text { Nivolumab } \\
\text { Pembrolizumab } \\
\text { Camrelizumab } \\
\text { Sintilimab } \\
\text { Others }\end{array}$ & $\begin{array}{l}\text { II (1I.80\%) } \\
15(16.10 \%) \\
19(20.40 \%) \\
24(25.80 \%) \\
24(25.80 \%)\end{array}$ & $\begin{array}{c}4(9.10 \%) \\
9(20.50 \%) \\
10(22.70 \%) \\
9(20.50 \%) \\
12(27.30 \%)\end{array}$ & $\begin{array}{c}7(14.30 \%) \\
6(12.20 \%) \\
9(18.40 \%) \\
15(30.60 \%) \\
12(24.50 \%)\end{array}$ & 0.607 \\
\hline
\end{tabular}

Notes: Others: Atezolizumab, Avelumab, Tislelizumab, Sugemalimab, Toripalimab ang HLXI0. *Indicates statistically significant P-values.

Abbreviations: PS, performance status; ICI, immune checkpoint inhibitor; CRP, C-reactive protein; LDH, lactate dehydrogenase; NLR, neutrophil-to-lymphocyte ratio. 
Table 2 Summary of Response in Study Population, ICI Monotherapy Subgroup and ICI Combination Therapy Group

\begin{tabular}{|c|c|c|c|c|c|}
\hline & & \multirow[t]{2}{*}{ Total $\mathbf{N}=93$} & \multicolumn{2}{|c|}{ Cavity and/or Necrosis } & \multirow[t]{2}{*}{$p$-value } \\
\hline & & & Positive $N=44$ & Negative $N=49$ & \\
\hline \multicolumn{6}{|l|}{ Objective response } \\
\hline Monotherapy & $N=39$ & 9 (23.1\%) & $2(10.0 \%)$ & 7 (36.8\%) & $0.047 *$ \\
\hline Combination therapy & $N=54$ & $30(55.6 \%)$ & $13(44.8 \%)$ & 17 (68.0\%) & 0.088 \\
\hline Total & $N=93$ & 39 (4I.9\%) & $15(30.6 \%)$ & 24 (54.5\%) & $0.020 *$ \\
\hline
\end{tabular}

Note: *Indicates statistically significant p-values.

summed diameters of all measurable tumor lesions, $\mathrm{LDH}$, and NLR, ROC curves also failed to identify any clinically significant threshold to predict the response to ICIs (Supplement Figure 1).

\section{Survival Analysis}

During the median follow-up of 20 weeks (range, 2136.6 weeks), a total of 73 events of progression or death were recorded. The univariate Cox proportional hazard analysis identified brain metastases, LDH > 250 IU/L, intrapulmonic necrosis or cavity, and ICI monotherapy as predictor candidates for a shorter PFS of advanced LSCC patients even after ICI treatment (Table 4). Accordingly, PFS of non-necrosis patients was significantly extended compared to the necrosis or cavity-positive group (median PFS, 26.7 weeks vs 16.0 weeks, $P=0.004$ ), with the similar results observed in the monotherapy (15.0 weeks vs 7.9 weeks, $P=0.012)$ and combination (81.9 weeks vs 21.6 weeks, $P=0.024)$ subgroups (Figure 1). The multivariate Cox analysis further confirmed brain metastases, ICI monotherapy, and intrapulmonic necrosis or cavity as risk predictors for a shorter PFS (HR:1.729, 95\% CI:1.203-2.484, $\mathrm{P}=0.003$ ) (Table 4).

\section{Discussion}

This study demonstrates that intrapulmonic necrosis or cavity present on baseline $\mathrm{CT}$ is related to low response to ICIs and poor PFS of advanced LSCC patients. Although this phenomenon is rather common among lung cancer patients, the present study, to the best of our knowledge, is the first to provide more information on the association of this basic character with ICI efficacy and patient prognosis in a real-world setting.

Predictive biomarkers for immune-checkpoint blockade (ICB) are being extensively investigated for personalized therapy. PD-L1 TPS has been recognized as a wellestablished biomarker for response to PD-(L)1 inhibitor in

Table 3 Univariable and Multivariable Analyses of Objective Response Rate

\begin{tabular}{|c|c|c|c|c|}
\hline & \multicolumn{2}{|c|}{ Univariable Model } & \multicolumn{2}{|c|}{ Multivariable Model } \\
\hline & OR $(95 \% \mathrm{Cl})$ & $p$-value & OR (95\% CI) & $p$-value \\
\hline Sum of tumor lesion diameters $>67.7 \mathrm{~cm}^{\dagger}$ & $0.742(0.322-1.710)$ & 0.484 & & \\
\hline $\mathrm{LDH}>250 \mathrm{IU} / \mathrm{L}$ & $1.925(0.705-5.258)$ & 0.202 & & \\
\hline$N L R>3$ & $1.316(0.576-3.006)$ & 0.515 & & \\
\hline Cavity and/or necrosis & $2.720(1.163-6.359)$ & $0.021^{*}$ & $4.042(1.149-10.908)$ & $0.006 *$ \\
\hline PD-LI TPS $\geq 1 \%$ & $0.536(0.089-3.210)$ & 0.494 & & \\
\hline PD-LI TPS $\geq 50 \%$ & $0.686(0.128-3.683)$ & 0.661 & & \\
\hline Treatment line & & & & \\
\hline$\geq 3^{\text {rd }}$ (reference) & & $0.046 *$ & & \\
\hline $1^{\text {st }}$ & $0.339(0.145-0.796)$ & 0.013 & & \\
\hline $2^{\text {nd }}$ & $1.576(0.996-2.494)$ & 0.052 & & \\
\hline Combination therapy & $0.240(0.096-0.601)$ & $0.002 *$ & $0.285(0.102-0.799)$ & $0.017^{*}$ \\
\hline
\end{tabular}

Notes: ${ }^{\dagger} 67.7 \mathrm{~cm}$ represented the mean of the sum of tumor lesion diameters in all LSCC patients. *Indicates statistically significant p-values. Abbreviations: $\mathrm{Cl}$, confidence interval; $\mathrm{OR}$, odd ratio. 
Table 4 Univariable and Multivariable Analyses of Progression Free Survival

\begin{tabular}{|c|c|c|c|c|}
\hline & \multicolumn{2}{|c|}{ Univariable Model } & \multicolumn{2}{|c|}{ Multivariable Model } \\
\hline & HR $(95 \% \mathrm{Cl})$ & $p$-value & HR $(95 \% \mathrm{Cl})$ & $p$-value \\
\hline Brain metastases & $1.855(1.117-3.078)$ & $0.017^{*}$ & $2.53 I(I .47 I-4.356)$ & $<0.00 I^{*}$ \\
\hline Liver metastases & $1.466(0.926-2.319)$ & 0.103 & & \\
\hline Bone metastases & $1.256(0.870-1.813)$ & 0.224 & & \\
\hline $\mathrm{LDH}>250 \mathrm{IU} / \mathrm{L}$ & $1.473(1.011-2.145)$ & $0.044^{*}$ & $1.436(0.977-2.112)$ & 0.066 \\
\hline NLR $>3$ & $1.169(0.842-1.624)$ & 0.350 & & \\
\hline PD-LI TPS $\geq 1 \%$ & $1.086(0.618-1.911)$ & 0.774 & & \\
\hline PD-LI TPS $\geq 50 \%$ & $1.126(0.590-2.150)$ & 0.720 & & \\
\hline Cavity and/or necrosis & $1.644(1.180-2.346)$ & $0.004 *$ & $1.729(1.203-2.484)$ & $0.003 *$ \\
\hline Combination therapy & $0.517(0.372-0.719)$ & $<0.00 I^{*}$ & $0.417(0.292-0.595)$ & $<0.00 I^{*}$ \\
\hline Sum of tumor lesion diameters $>67.7 \mathrm{~cm}^{*}$ & $1.09 \mid(0.78|-| .5 \mid 7)$ & 0.605 & & \\
\hline
\end{tabular}

Note: *Indicates statistically significant $\mathrm{p}$-values.

Abbreviations: $\mathrm{Cl}$, confidence interval; $\mathrm{HR}$, hazard ratio.

NSCLC. However, a large-scale, retrospective, real-world study of 1460 patients recently argued that PD-L1 could not be an appropriate predictive biomarker for ICI use in NSCLC with squamous histology. ${ }^{13}$ Similar results were observed in our study and other publications. For example, high PD-L1 expression correlated with tumor necrosis has been confirmed in lung adenocarcinoma, ${ }^{25}$ pulmonary pleomorphic carcinoma, ${ }^{26}$ and oral squamous cell carcinoma. ${ }^{27}$ The relationship of PD-L1 expression with other subtypes of lung squamous carcinomas, including LSCC, has not been fully understood. And potential efficacy predictors for monitoring immunotherapy and estimating LSCC prognosis are a field wait for exploration. Preexisting studies on this topic have not reached a consensus on the predictive values of PD-L1 expression or TMB in studies of NSCLC patients receiving anti-PD-(L)1-based combination treatment. ${ }^{2,8}$ The present study offers new insight into a common imaging sign, baseline necrosis or cavity on $\mathrm{CT}$, whose predictive power has been observed in patients undergoing ICI mono and combination immunotherapy.

Amid a surge of biomarker and drug target studies, morphological characteristics on $\mathrm{CT}$ are rarely figured as important players for efficacy prediction of cancer treatment in advanced lung cancers, even NSCLC. Ma et al ascertained an association between pleura thickening/ indentation of stage I lung adenocarcinoma and its prognosis. ${ }^{28}$ Choi et al reported that CT imaging characteristics at diagnosis were associated with clinical response to EGFR-tyrosine kinase inhibitors. ${ }^{29}$ Patients with NSCLC with interlobular septal thickening may poorly respond to nivolumab. ${ }^{30}$ Intratreatment radiographic assessments, particularly noninvasive functional imaging, can generate quantitative data on tumor dynamics that has been found to be associated with clinical response to and outcomes of precision cancer therapies. They are also useful tools for clinical response or prognostic stratification in patients undergoing immunotherapy. ${ }^{31}$ The combination of deep learning and $\mathrm{CT}$ imaging will be more efficient in screening biomarkers for efficacy prediction of novel ICIs, such as nivolumab, in NSCLC.

A retrospective analysis showed that lower tumor burden is associated with greater efficacy of PD-1/PD-L1 inhibitor monotherapy for patients with advanced NSCLC. ${ }^{32}$ We estimated the tumor burden using the baseline sum of diameters of all measurable tumor lesions, and the multivariate Cox analysis showed that it was not associated with PFS. Whether tumor burden is directly related to intrapulmonic necrosis or cavity is unknown. Therefore, more studies are needed to determine the mechanism. Tumor necrosis is usually induced by nutrient deficiency and the resultant hypoxia. ${ }^{14,15}$ Since many efforts to better understand how hypoxia and metabolic stress contribute to drug resistance were made, almost all findings have turned out to be inflammatory mechanisms. ${ }^{33-35}$ The latest cumulating retrospective analyses indicate that common inflammatory lab markers, such as LDH, CRP, NLR, and IL-6, are associated with the outcomes of advanced NSCLC patients undergoing ICIs. ${ }^{36,37}$ Of note, their associations are not as tight as those with baseline necrosis or cavity on $\mathrm{CT}$, which was confirmed as an independent predictor of 
A

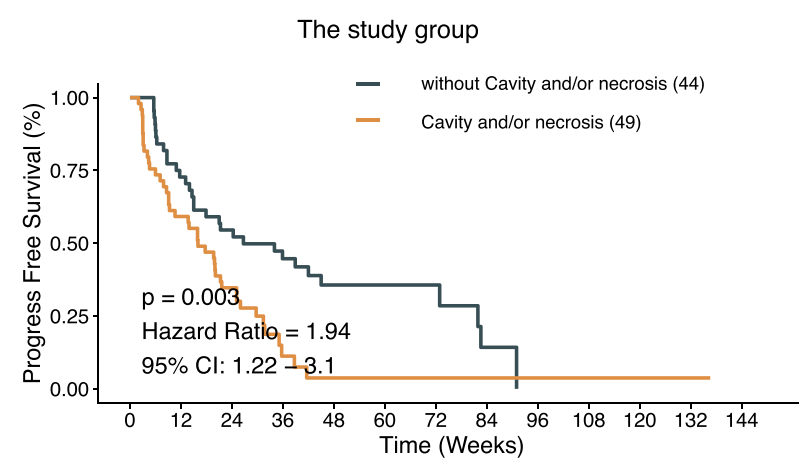

Number at risk

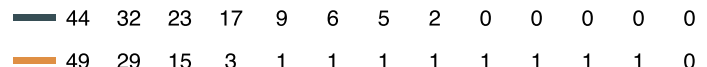

C

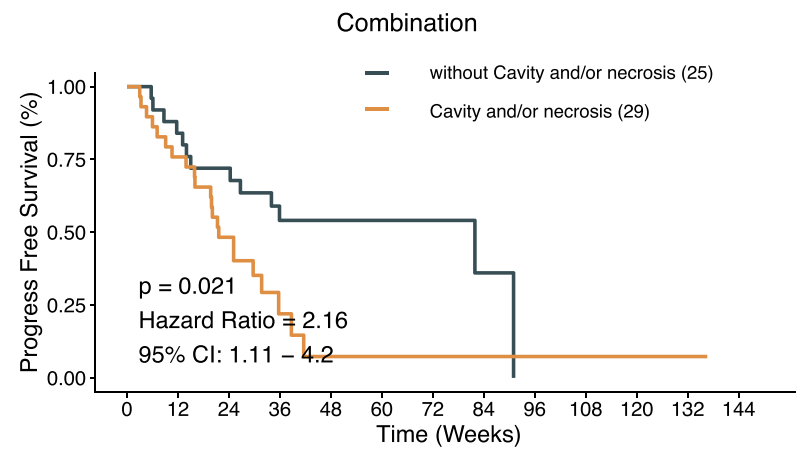

Number at risk

$\begin{array}{lllllllllllll}25 & 21 & 17 & 11 & 6 & 3 & 3 & 2 & 0 & 0 & 0 & 0 & 0 \\ 29 & 22 & 12 & 3 & 1 & 1 & 1 & 1 & 1 & 1 & 1 & 1 & 0\end{array}$

B

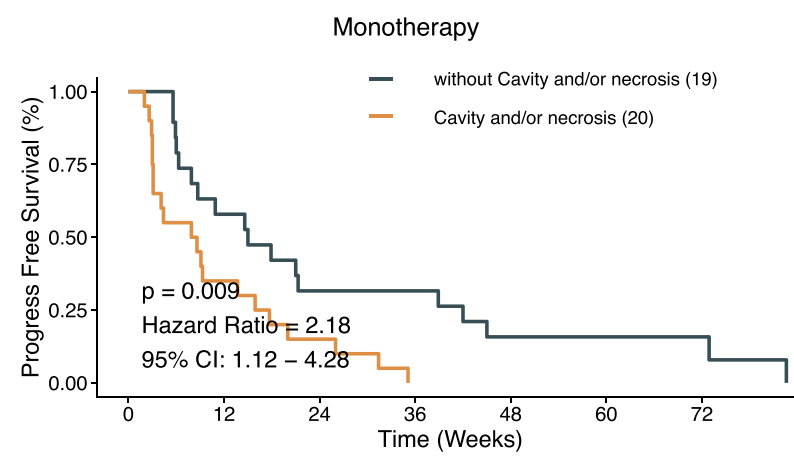

Number at risk

$\begin{array}{rrrrrrr}19 & 11 & 6 & 6 & 3 & 3 & 2 \\ -20 & 7 & 3 & 0 & 0 & 0 & 0\end{array}$

Figure I Progression free survival of advanced LSCC patients with versus without intrapulmonic necrosis or cavity on baseline CT scan. (A) PFS in the study population. (B) $\mathrm{PFS}$ in the $\mathrm{ICl}$ monotherapy subgroup. (C) PFS in the $\mathrm{ICl}$ combination subgroup.

response to ICI treatment. One possible reason is that serum levels of these inflammatory markers dramatically fluctuate during the treatment, compared to morphological features like necrosis or cavity. Future studies can elucidate the precise mechanisms governing the relationship between necrosis or cavity and patient prognosis after ICI treatment.

Available evidence on the prognostic value of tumor necrosis in LSCC, primarily provided by retrospective studies, is limited and remains weak. ${ }^{38-41}$ And its detection largely relies on CT scan rather than histological examination as biopsy specimen does not necessarily incorporate the necrotic-core component and cannot outline the morphology of necrosis as clearly as CT imaging. Besides, necrosis is detectable on CT only when it grows to a certain size. Interestingly, we found that necrosis or cavity at baseline was closely associated with the summed diameters of all measurable tumor lesions. However, the latter was not associated with clinical response to ICI treatment. Overall, although we ascertained intrapulmonic necrosis or cavity on baseline CT as an efficacy predictor, this conclusion needs further validation by prospective, non-ICI treatment controlled, multicenter trials.

There are some limitations concerning study design and patient stratification. Limited by the retrospective nature, the sample size of the current study, particularly in the cohort matched for baseline characteristics, was relatively small. Despite ICI treatment, patients included also received chemotherapy or radiotherapy. Unlike chronic necrosis, treatment-related acute necroptosis (referring to 
regulated cell death with necrotic morphology) is more immunogenetic and favors anti-tumor immunoactivity. ${ }^{14,15}$ In our study, patients of this kind were not excluded due to insufficient medical records and limited sample size. The potential influence of treatment-related necroptosis on immune response remains to be determined.

In conclusion, LSCC patients with intrapulmonic necrosis or cavity on baseline $\mathrm{CT}$ may respond poorly to anti-PD-(L)1-treatment, whether mono or combination therapy. This finding warrants further validations by rigorously designed prospective trials, leading to an improved understanding of how tumor necrosis is involved in LSCC progression.

\section{Ethics Approval and Consent to Participate}

This study was conducted in accordance with the declaration of Fujian Cancer Hospital Clinical Research Ethics Committee approved this retrospective study. The requirement for informed consent was waived due to the retrospective nature of the study, and no personal information was disclosed. This study was conducted in accordance with the Declaration of Helsinki.

\section{Author Contributions}

All authors made a significant contribution to the work reported, whether that is in the conception, study design, execution, acquisition of data, analysis and interpretation, or in all these areas; took part in drafting, revising or critically reviewing the article; gave final approval of the version to be published; have agreed on the journal to which the article has been submitted; and agree to be accountable for all aspects of the work.

\section{Funding}

The funding agencies had no role in the study design, data collection and analysis, decision to publish, or manuscript preparation. This work was supported by the National Natural Science Foundation of China (grant 82072565), the Fujian Provincial Health Systemic Innovation Project (grant 2020CXA010), the Fujian Provincial Health Commission (Grant number 2020QNA014), Beijing Xisike Clinical Oncology Research Foundation (grant Y-2019AZMS-0434), Fujian Provincial Natural Science Foundation (grant 2020J011119).

\section{Disclosure}

The authors declare that they have no conflicts of interest for this work.

\section{References}

1. Remon J, Passiglia F, Ahn MJ, et al. Immune checkpoint inhibitors in thoracic malignancies: review of the existing evidence by an IASLC expert panel and recommendations. J Thorac Oncol. 2020;15 (6):914-947.

2. Lantuejoul S, Sound-Tsao M, Cooper WA, et al. PD-L1 testing for lung cancer in 2019: perspective from the IASLC Pathology Committee. J Thorac Oncol. 2020;15(4):499-519. doi:10.1016/j. jtho.2019.12.107

3. Mok TSK, Wu Y, Kudaba I, et al. Pembrolizumab versus chemotherapy for previously untreated, PD-L1-expressing, locally advanced or metastatic non-small-cell lung cancer (KEYNOTE-042): a randomised, open-label, controlled, Phase 3 trial. Lancet. 2019;393(10183):1819-1830. doi:10.1016/S0140-6736(18)32409-7

4. Leighl NB, Hellmann MD, Hui R, et al. Pembrolizumab in patients with advanced non-small-cell lung cancer (KEYNOTE-001): 3-year results from an open-label, Phase 1 study. Lancet Respir Med. 2019;7 (4):347-357. doi:10.1016/S2213-2600(18)30500-9

5. Reck M, Rodríguez-Abreu D, Robinson AG, et al. Pembrolizumab versus chemotherapy for PD-L1-positive non-small-cell lung cancer. New Engl $J$ Med. 2016;375(19):1823-1833. doi:10.1056/ NEJMoa1606774

6. Garon EB, Rizvi NA, Hui R, et al. Pembrolizumab for the treatment of non-small-cell lung cancer. $N$ Engl $J$ Med. 2015;372 (21):2018-2028. doi:10.1056/NEJMoa1501824

7. Osipov A, Lim SJ, Popovic A, et al. Tumor mutational burden, toxicity, and response of immune checkpoint inhibitors targeting PD(L)1, CTLA-4, and combination: a meta-regression analysis. Clin Cancer Res. 2020;26(18):4842-4851. doi:10.1158/1078-0432. CCR-20-0458

8. Sha D, Jin Z, Budczies J, et al. Tumor mutational burden as a predictive biomarker in solid tumors. Cancer Discov. 2020;10 (12):1808-1825. doi:10.1158/2159-8290.CD-20-0522

9. Marabelle A, Le DT, Ascierto PA, et al. Efficacy of pembrolizumab in patients with noncolorectal high microsatellite instability/mismatch repair-deficient cancer: results from the Phase II KEYNOTE-158 Study. J Clin Oncol. 2020;38(1):1-10. doi:10.1200/JCO.19.02105

10. Petrelli F, Ghidini M, Ghidini A, et al. Outcomes following immune checkpoint inhibitor treatment of patients with microsatellite instability-high cancers: a systematic review and meta-analysis. JAMA Oncol. 2020;6(7):1068. doi:10.1001/jamaoncol.2020.1046

11. Overman MJ, McDermott R, Leach JL, et al. Nivolumab in patients with metastatic DNA mismatch repair-deficient or microsatellite instability-high colorectal cancer (CheckMate 142): an open-label, multicentre, Phase 2 study. Lancet Oncol. 2017;18(9):1182-1191. doi:10.1016/S1470-2045(17)30422-9

12. Redman MW, Papadimitrakopoulou VA, Minichiello K, et al. Biomarker-driven therapies for previously treated squamous non-small-cell lung cancer (Lung-MAP SWOG S1400): a biomarker-driven master protocol. Lancet Oncol. 2020;21 (12):1589-1601. doi:10.1016/S1470-2045(20)30475-7

13. Doroshow D, Wei W, Zugazagoitia J, et al. PD-L1 tumor proportion score and clinical benefit from first-line pembrolizumab in patients with advanced nonsquamous versus squamous non-small cell lung cancer (NSCLC). J Clin Oncol. 2020;38:9539. doi:10.1200/ JCO.2020.38.15_suppl.9539

14. Liu Z, Jiao D. Necroptosis, tumor necrosis and tumorigenesis. Cell Stress. 2019;4(1):1-8. doi:10.15698/cst2020.01.208 
15. Green DR. The coming decade of cell death research: five riddles Cell. 2019;177(5):1094-1107. doi:10.1016/j.cell.2019.04.024

16. Brown JM, Wilson WR. Exploiting tumour hypoxia in cancer treatment. Nat Rev Cancer. 2004;4(6):437-447. doi:10.1038/nrc1367

17. Gatenby RA, Gillies RJ. Why do cancers have high aerobic glycolysis? Nat Rev Cancer. 2004;4(11):891-899. doi:10.1038/ nrc1478

18. Lotfi R, Kaltenmeier C, Lotze MT, et al. Until death do us part: necrosis and oxidation promote the tumor microenvironment. Transfus Med Hemother. 2016;43(2):120-132. doi:10.1159/ 000444941

19. Werfel TA, Elion DL, Rahman B, et al. Treatment-induced tumor cell apoptosis and secondary necrosis drive tumor progression in the residual tumor microenvironment through MerTK and IDO1. Cancer Res. 2019;79(1):171-182. doi:10.1158/0008-5472.CAN-18-1106

20. Hijaze N, Ledersnaider M, Simanovich E, et al. Inducing regulated necrosis and shifting macrophage polarization with anti-EMMPRIN antibody (161-pAb) and complement factors. J Leukoc Biol. 2020. doi:10.1002/JLB.3A0520-333R

21. Atanasov G, Dino K, Schierle K, et al. Angiogenic inflammation and formation of necrosis in the tumor microenvironment influence patient survival after radical surgery for de novo hepatocellular carcinoma in non-cirrhosis. World J Surg Oncol. 2019;17(1):217. doi:10.1186/s12957-019-1756-8

22. Eil R, Vodnala SK, Clever D, et al. Ionic immune suppression within the tumour microenvironment limits $\mathrm{T}$ cell effector function. Nature. 2016;537(7621):539-543. doi:10.1038/nature19364

23. Eisenhauer EA, Therasse P, Bogaerts J, et al. New response evaluation criteria in solid tumours: revised RECIST guideline (version 1.1). Eur $J$ Cancer. 2009;45(2):228-247. doi:10.1016/j. ejca.2008.10.026

24. Hansell DM, Bankier AA, MacMahon H, et al. Fleischner Society: glossary of terms for thoracic imaging. Radiology. 2008;246 (3):697-722. doi:10.1148/radiol.2462070712

25. Reiniger L, Téglási V, Pipek $\mathrm{O}$, et al. Tumor necrosis correlates with PD-L1 and PD-1 expression in lung adenocarcinoma. Acta Oncol. 2019;58(8):1087-1094. doi:10.1080/0284186X.2019.1598575

26. Chang YL, Yang CY, Lin MW, et al. High co-expression of PD-L1 and HIF-1 $\alpha$ correlates with tumour necrosis in pulmonary pleomorphic carcinoma. Eur J Cancer. 2016;60:125-135. doi:10.1016/j. ejca.2016.03.012

27. Chen TC, Wu CT, Wang CP, et al. Associations among pretreatment tumor necrosis and the expression of HIF-1 $\alpha$ and PD-L1 in advanced oral squamous cell carcinoma and the prognostic impact thereof. Oral Oncol. 2015;51(11):1004-1010. doi:10.1016/j.oraloncology.2015. 08.011

28. Ma J, Yang YL, Wang Y, et al. Relationship between computed tomography morphology and prognosis of patients with stage I non-small cell lung cancer. Onco Targets Ther. 2017;10:2249-2256. doi:10.2147/OTT.S114960
29. Choi CM, Kim MY, Lee JC, et al. Advanced lung adenocarcinoma harboring a mutation of the epidermal growth factor receptor: CT findings after tyrosine kinase inhibitor therapy. Radiology. 2014;270 (2):574-582. doi:10.1148/radiol.13121824

30. Minemura $\mathrm{H}$, Moriya $\mathrm{H}$, Imai $\mathrm{H}$, et al. Prognostic value of morphological characteristics assessed by $\mathrm{CT}$ scan in patients with non-small cell lung cancer treated with nivolumab. Thorac Cancer. 2020;11 (12):3521-3527. doi:10.1111/1759-7714.13695

31. Xu Y, Hosny A, Zeleznik R, et al. Deep learning predicts lung cancer treatment response from serial medical imaging. Clin Cancer Res. 2019;25(11):3266-3275. doi:10.1158/1078-0432.CCR-18-2495

32. Miyawaki T, Kenmotsu H, Mori K, et al. Association between clinical tumor burden and efficacy of immune checkpoint inhibitor monotherapy for advanced non-small-cell lung cancer. Clin Lung Cancer. 2020;21(5):e405-e414. doi:10.1016/j.cllc.2020.02.012

33. Galdiero MR, Marone G, Mantovani A. Cancer inflammation and cytokines. Csh Perspect Biol. 2018;10(8):a28662.

34. Diakos CI, Charles KA, McMillan DC, et al. Cancer-related inflammation and treatment effectiveness. Lancet Oncol. 2014;15(11):e493e503. doi:10.1016/S1470-2045(14)70263-3

35. Greten FR, Grivennikov SI. Inflammation and cancer: triggers, mechanisms, and consequences. Immunity. 2019;51(1):27-41.

36. Pavan A, Calvetti L, Dal Maso A, et al. Peripheral blood markers identify risk of immune-related toxicity in advanced non-small cell lung cancer treated with immune-checkpoint inhibitors. Oncologist. 2019;24(8):1128-1136. doi:10.1634/theoncologist.2018-0563

37. Duchemann B, Remon J, Naigeon M, et al. Integrating circulating biomarkers in the immune checkpoint inhibitor treatment in lung cancer. Cancers. 2020;12(12):3625. doi:10.3390/cancers 12123625

38. Gürel D, Ulukuş Ç, Karaçam V, et al. The prognostic value of morphologic findings for lung squamous cell carcinoma patients. Pathology Res Practice. 2016;212(1):1-9. doi:10.1016/j. prp.2015.10.006

39. Chen S, Gao C, Du Q, et al. A prognostic model for elderly patients with squamous non-small cell lung cancer: a population-based study. $J$ Transl Med. 2020;18(1). doi:10.1186/s12967-020-02606-3

40. Koike Y, Aokage K, Ikeda K, et al. Machine learning-based histological classification that predicts recurrence of peripheral lung squamous cell carcinoma. Lung Cancer. 2020;147:252-258. doi:10.1016/ j.lungcan.2020.07.011

41. Park SY, Lee H, Jang $\mathrm{H}$, et al. Tumor necrosis as a prognostic factor for stage IA non-small cell lung cancer. Ann Thorac Surg. 2011;91 (6):1668-1673. doi:10.1016/j.athoracsur.2010.12.028

\section{Publish your work in this journal}

Cancer Management and Research is an international, peer-reviewed open access journal focusing on cancer research and the optimal use of preventative and integrated treatment interventions to achieve improved outcomes, enhanced survival and quality of life for the cancer patient.
The manuscript management system is completely online and includes a very quick and fair peer-review system, which is all easy to use. Visit http://www.dovepress.com/testimonials.php to read real quotes from published authors. 